\title{
Determination of Rheological Parameters of Polyvinylchloride at Different Temperatures
}

\author{
A.S. Chepurnenko ${ }^{1, \mathrm{a}}$, V.I. Andreev ${ }^{2, \mathrm{~b}}$, A.N. Beskopylny ${ }^{1, \mathrm{c}}$ and B.M. Jazyev ${ }^{1, \mathrm{~d}}$ \\ ${ }^{1}$ Rostov-on-Don State University of Civil Engineering, Rostov-on-Don, Russia \\ ${ }^{2}$ Moscow State University of Civil Engineering, Yaroslavskoye sh., 26, 129337 Moscow, Russia \\ anton_chepurnenk@mail.ru, ${ }^{\mathrm{a}}$ asv@mgsu.ru, ${ }^{\mathrm{c}}$ besk-an@yandex.ru, ${ }^{\mathrm{d}} 277588 @$ rambler.ru
}

\begin{abstract}
The paper proposes the method of determining the relaxation constants of the material included in the nonlinear equation of Maxwell-Gurevich. We performed the processing of relaxation curves of recycled polyvinylchloride (PVC) at different temperatures and investigated the effect of temperature on the value of the relaxation parameters. For each parameter, we have chosen empirical formula describing its dependence on temperature.
\end{abstract}

Keywords: recycled polyvinylchloride, relaxation, Maxwell-Gurevich law, high elasticity modulus, relaxation viscosity, high elastic deformations

\section{Introduction}

For polymers of mesh and linear structure, the connection between the creep strain and stress is set by nonlinear equation of Maxwell-Gurevich [1]. In the case of a uniaxial stress state, it has the form [2]:

$$
\frac{\partial \varepsilon^{*}}{\partial t}=\frac{f^{*}}{\eta^{*}},
$$

where $\varepsilon^{*}$ - highly elastic deformation, $f^{*}$ - stress function, $\eta^{*}$ - relaxation viscosity.

The stress function and relaxation viscosity are calculated as follows:

$$
f^{*}=\sigma-E_{\infty} \varepsilon^{*} ; \eta^{*}=\eta_{0}^{*} \exp \left(-\frac{\left|f^{*}\right|}{m^{*}}\right) \text {. }
$$

Here $\sigma$-stress, $E_{\infty}$ - high elasticity modulus, $\eta_{0}^{*}-$ the initial relaxation viscosity, $m^{*}$ - velocity module. 


\section{Solution of Problem}

We consider the method for determining the rheological parameters using the relaxation curves and establish the dependences of each parameter on the temperature on the example of recycled PVC. The results of tests on the stress relaxation of the $\mathrm{PVC}$ at a relative deformation $\varepsilon=3 \%$, taken from [3], are given in table 1 .

The total deformation $\varepsilon$, which in the process of relaxation remains constant, is the sum of the elastic and highly elastic deformation:

$$
\varepsilon=\frac{\sigma(t)}{E}+\varepsilon^{*}(t)
$$

where $E$ - instantaneous modulus of elasticity.

Since in the initial moment of time there is no highly elastic deformation, then we can immediately find the value of $E$ :

$$
E=\sigma(0) / \varepsilon \text {. }
$$

TABLE 1. CHANGE OF STRESSES IN TIME AT DIFFERENT TEMPERATURES

\begin{tabular}{|l|c|c|c|c|c|c|c|c|c|c|c|}
\hline \multicolumn{2}{|l|}{$\mathrm{t}, \min$} & 0 & 3 & 6 & 15 & 30 & 45 & 60 & 90 & 120 & 180 \\
\hline \multirow{4}{*}{$\sigma}$, & $20{ }^{\circ} \mathrm{C}$ & 44.4 & 40.8 & 39.8 & 38.8 & 38 & 37.7 & 37.3 & 36.9 & 36.4 & 35.6 \\
\cline { 2 - 13 } $\mathrm{M}$ & $30{ }^{\circ} \mathrm{C}$ & 43.4 & 36.7 & 35.7 & 34.3 & 33 & 32.2 & 31.9 & 30.8 & 30.3 & 29.2 \\
\cline { 2 - 12 } & $40{ }^{\circ} \mathrm{C}$ & 39.3 & 32.9 & 31.4 & 29 & 27 & 25.9 & 24.9 & 23.8 & 22.7 & 21.3 \\
\cline { 2 - 12 } $\mathrm{Pa}$ & $50{ }^{\circ} \mathrm{C}$ & 36.4 & 20.5 & 18.8 & 16.5 & 14.7 & 13.9 & 13 & 12.5 & 11.9 & 11.1 \\
\cline { 2 - 11 } & $60{ }^{\circ} \mathrm{C}$ & 33.4 & 15.4 & 13.4 & 10.6 & 8.61 & 7.63 & 6.95 & 6.02 & 5.42 & 5.05 \\
\cline { 2 - 11 } & $70{ }^{\circ} \mathrm{C}$ & 23.4 & 5.06 & 4.05 & 2.84 & 2.48 & 2.16 & 2 & 1.85 & 1.58 & 1.31 \\
\hline
\end{tabular}

If we know the modulus of elasticity, we can determine the highly elastic deformation at each time:

$$
\varepsilon^{*}(t)=\varepsilon-\frac{\sigma(t)}{E}
$$

Differentiating (5) with respect to time, we get:

$$
\frac{\partial \varepsilon^{*}}{\partial t}=-\frac{1}{E} \frac{\partial \sigma}{\partial t} \text {. }
$$

Next, the function $\sigma(t)$ must be numerically differentiated with respect to $t$, to find the value

$$
v_{\varepsilon}=\frac{\partial \varepsilon^{*}}{\partial t}
$$

at any moment of time. To do this, we use the method of undetermined coefficients.

At the end of relaxation process, the growth rate of highly elastic deformation is equal to zero. Using this condition we can obtain the value of $E_{\infty}$ :

$$
v_{\varepsilon}=\frac{\partial \varepsilon^{*}}{\partial t}=\frac{f^{*}}{\eta^{*}}=0 \Rightarrow f^{*}=\sigma-E_{\infty} \varepsilon^{*}=0 \Rightarrow E_{\infty}=\frac{\sigma(\infty)}{\varepsilon^{*}(\infty)} .
$$


If we know the value of $E_{\infty}$, we can find at any given time values of $f^{*}$ and $\eta^{*}=f^{*} / v_{\varepsilon}$. Then we take a logarithm of expression for $\eta^{*}$ in (2):

$$
\ln \eta^{*}=\ln \left(\eta_{0}^{*} e^{-\frac{\left|f^{*}\right|}{m^{*}}}\right)=\ln \eta_{0}^{*}-\frac{\left|f^{*}\right|}{m^{*}}
$$

Expression (8) shows that the value of $y=\ln \eta^{*}$ must be linearly dependent on the value of $x=\left|f^{*}\right|$, i.e.

$$
y=a x+b, \text { where } a=-1 / m^{*}, b=\ln \eta_{0}^{*} \text {. }
$$

Having multiple values of $\ln \eta^{*}$, corresponding to different values of $\left|f^{*}\right|$, we can obtain coefficients $a$ and $b$ by the method of least squares, and then find the velocity modulus $m^{*}$ and the initial relaxation viscosity $\eta_{0}^{*}$.

\section{Results and Discussion}

From the tests on stress relaxation, we can define all the parameters in the equation of Maxwell-Gurevich. The results of processing of experimental data are summarized in table 2.

TABLE 2. ELASTIC AND RELAXATION PARAMETERS OF THE RECYCLED PVC AT DIFFERENT TEMPERATURES

\begin{tabular}{|c|c|c|c|c|c|c|}
\hline$T,{ }^{\circ} \mathrm{C}$ & 20 & 30 & 40 & 50 & 60 & 70 \\
\hline$E, \mathrm{MPa}$ & 1480 & 1450 & 1310 & 1210 & 1110 & 780 \\
\hline$E_{\infty}, \mathrm{MPa}$ & 5990 & 2975 & 1550 & 532 & 198 & 46.3 \\
\hline$m^{*}, \mathrm{MPa}$ & 12.6 & 12.1 & 13.9 & 11.2 & 11.8 & 7.76 \\
\hline$\eta_{0}^{*} \cdot 10^{-5}, \mathrm{MPa} \cdot \min$ & 9.06 & 5.17 & 2.81 & 0.891 & 0.48 & 0.256 \\
\hline
\end{tabular}

Graphs of change of the elastic modulus $E$, high elasticity modulus $E_{\infty}$, initial relaxation viscosity $\eta_{0}^{*}$ and the velocity modulus $m^{*}$ depending on the temperature are represented respectively in Figures 1-4. 


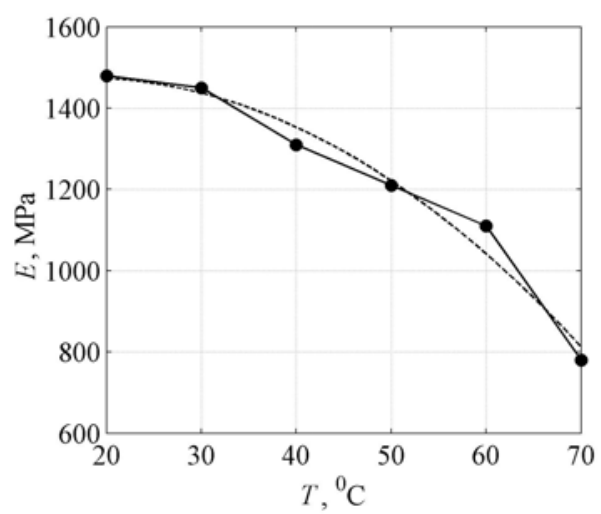

Fig. 1. Dependence of elastic modulus on temperature

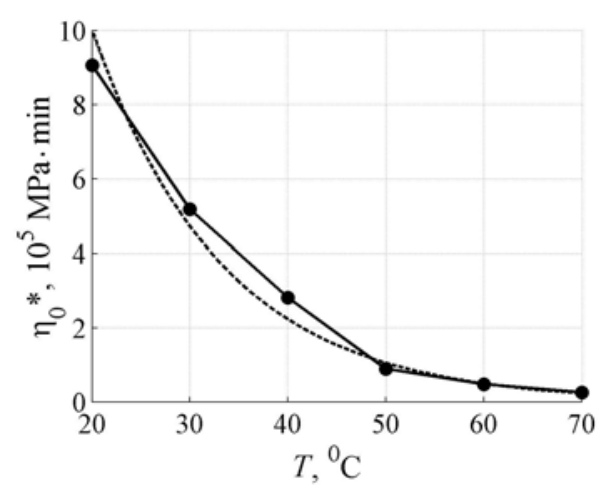

Fig. 3. Dependence of the initial relaxation viscosity on temperature

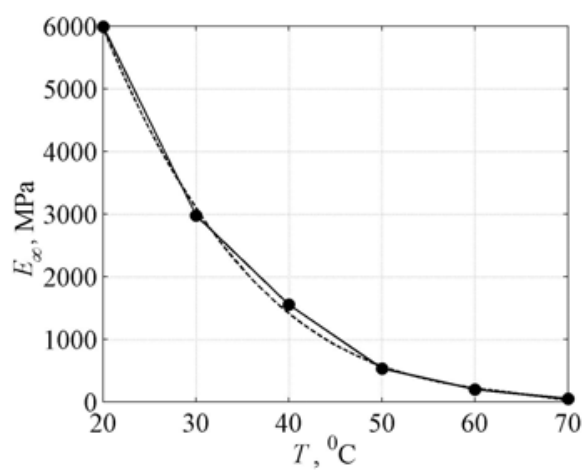

Fig. 2. Dependence of high elasticity modulus on temperature

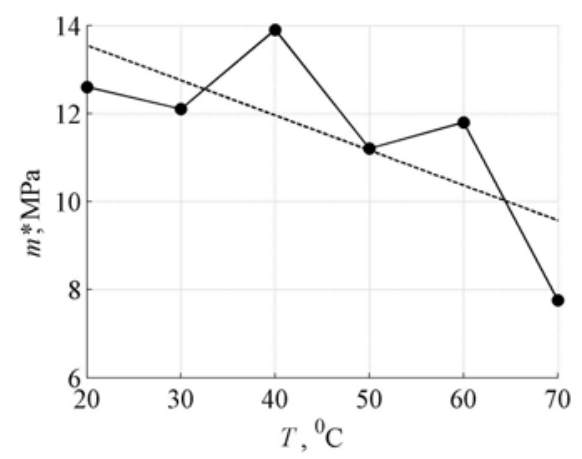

Fig. 4. Dependence of the velocity modulus on temperature

Figures $1-3$ show that the elastic modulus, high elasticity modulus and the initial relaxation viscosity of PVC are highly dependent on temperature. The dashed lines in each graph show the approximate curves. The dependence of elastic modulus on temperature is of the form:

$$
E(T)=-0.2393 T^{2}+8.3357 T+14026,[\mathrm{MPa}] ., E(T)=
$$

where $T$ - temperature in degrees centigrade.

Dependence of high elasticity modulus on temperature can be approximated as follows:

$$
E_{\infty}(T)=-0.0575 T^{3}+11.095 T^{2}-732 T+16618,[\mathrm{MPa}] .
$$

The relationship between the initial relaxation viscosity and temperature is accurately approximated by an exponential dependence:

$$
\eta_{0}^{*}(T)=44.78 \cdot 10^{5} \exp (-0.075 T),[\mathrm{MPa} \cdot \min ] .
$$

It is necessary to note that for polymers PMMA and EDT dependences of the initial relaxation viscosity on temperature are also exponential [4]. 
The magnitude of the velocity modulus at a temperature from 20 to 60 degrees is approximately constant and at 70 degrees slightly decreases. It is proposed to approximate $m^{*}(T)$ by a linear function:

$$
m^{*}(T)=-0.0794 T+15.134,[\mathrm{MPa}]
$$

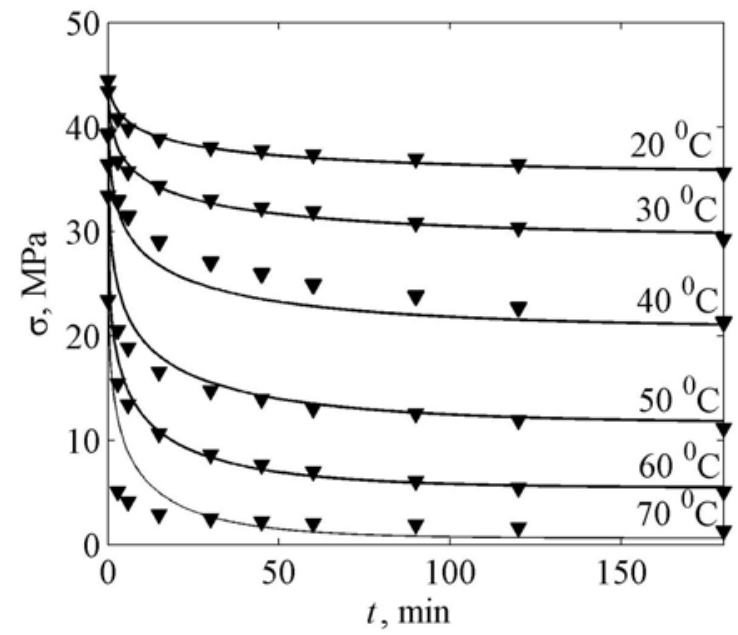

Fig. 5. Theoretical and experimental relaxation curves

The solid lines on Figure 5 show relaxation curves calculated on the basis of the Maxwell-Gurevich law and dependencies (9) - (12). The triangular markers correspond to the experimental values of the stress. At $T=20{ }^{\circ} \mathrm{C}, 30{ }^{\circ} \mathrm{C}$ and $60{ }^{\circ} \mathrm{C}$ agreement with the experimental data is very good, at the rest values of temperature - is satisfactory.

\section{Conclusions}

Determination of the viscoelastic properties of materials is a primary and a very important component in the design of structures made of polymers and composite materials based on polymers. The paper presents a simple method of determining the characteristics using the experimental data on the stress relaxation at various temperatures. Usage as a physical law of Maxwell-Gurevich equation (generalized equation of Maxwell) is due to the numerous studies of authors in the field of analysis of polymeric structures in the different modes [5 $10]$.

\section{Acknowledgement}

Scientific work is realize under support of Ministry education and science Russian Federation (grant of President Russian Federation No14.Z57.14.6545-SS) 


\section{References}

1. G.I. Gurevich, Deformability of substances and propagation of seismic waves, Nauka, Moscow, 1974.

2. A.L. Rabinovich, Introduction to the mechanics of reinforced polymers, Nauka, Moscow, 1970.

3. E.V. Solovieva, A.A. Askadskii, M.N. Popova, Study of relaxation properties of the primary and secondary PVC, Plastics. 2 (2013) 54-62.

4. B.M. Yazyev, V.I. Andreev, R.A. Turusov, Some problems and methods of mechanics of viscoelastic polymer medium, monograph, RGSU, Rostov-on-Don, 2009.

5. V.I. Andreev, About the Unloading in Elastoplastic Inhomogeneous Bodies, Applied Mechanics and Materials. 353-356( 2013) 1267-1270.

6. V.I. Andreev, A.S. Chepurnenko, B.M. Yazyev, On the Bending of a Thin Plate at Nonlinear Creep, Advanced Materials Research. 900 (2014) 707-710.

7. V.I. Andreev, A.S. Chepurnenko, B.M. Yazyev, Energy Method in the Calculation Stability of Compressed Polymer Rods Considering Creep, Advanced Materials Research. 1004-1005 (2014) 257-260.

8. V.I. Andreev, A.S. Chepurnenko, B.M. Yazyev, Axisymmetric bending of a flexible circular plate in the creep. Vestnik MGSU [Bulletin of MGSU] 5 (2014) 16-24.

9. R.A. Turusov, V.I. Andreev, Thermal stresses and relaxation phenomena in polymer rods. Proceedings of the International Conference on Advanced Materials and Engineering Structural Technology (ICAMEST 2015), Qingdao, China , April 25-26, 2015 .

10. V.I. Andreev, R.A. Turusov, Nonlinear Modeling of the Kinetics of Thermal Stresses in the Polymer Rods, Proceedings of the International Conference on Advanced Materials and Engineering Structural Technology (ICAMEST 2015), Qingdao, China , April 25-26, 2015. 